\title{
FPV Vaccine CV301
}

National Cancer Institute

\section{Source}

National Cancer Institute. FPV Vaccine CV301. NCI Thesaurus. Code C141298.

A cancer vaccine consisting of a recombinant fowlpox viral (FPV) vector encoding both the two human tumor-associated antigens (TAAs) carcinoembryonic antigen (CEA) and mucin-1 (MUC-1), and TRICOM, which is comprised of the three human immuneenhancing co-stimulatory molecules B7-1, ICAM-1 and LFA-3, with potential immunostimulatory and antineoplastic activities. Upon subcutaneous administration following the administration of a proprietary modified vaccinia Ankara developed by Bavarian Nordic-based prime vaccine MVA-BN-CV301, the FPV vaccine CV301, which is used as a booster vaccine, activates a cytotoxic T-lymphocyte $(C T L)$ response ag ainst CEA- and MUC-1-expressing tumor cells. In addition, the CV301-dependent anti-tumor CT L response upregulates the expression of programmed cell death ligand 1 (PD-L1); therefore, when FPV-CV301 is combined with a programmed cell death 1 (PD-1) immune checkpoint inhibitor, the antitumor effect may be increased. The TAAs CEA and MUC-1 are overexpressed in a variety of cancers. 